Supplementary data for:

\title{
Improved hBN single crystal growth by adding carbon in the metal flux
}

Si-Yuan ZHANG, Kang XU, Xiao-Kang ZHAO, Zhi-Yong SHAO, Neng WAN*

SEU-FEI Nano Pico center, Key Laboratory of MEMS of Ministry of Education, School of Electronics Science and Engineering, Southeast University, 210096 Nanjing, People's Republic of China

This data includes:

EDS mapping images of the alloy ingot section at the carbon ratio of $0 \%$ (Fig. s1) and $1.8 \%$ (Fig. s2). The corresponding discussion is in the paper (page 10, paragraph 5).

*Correspondence: wn@ 

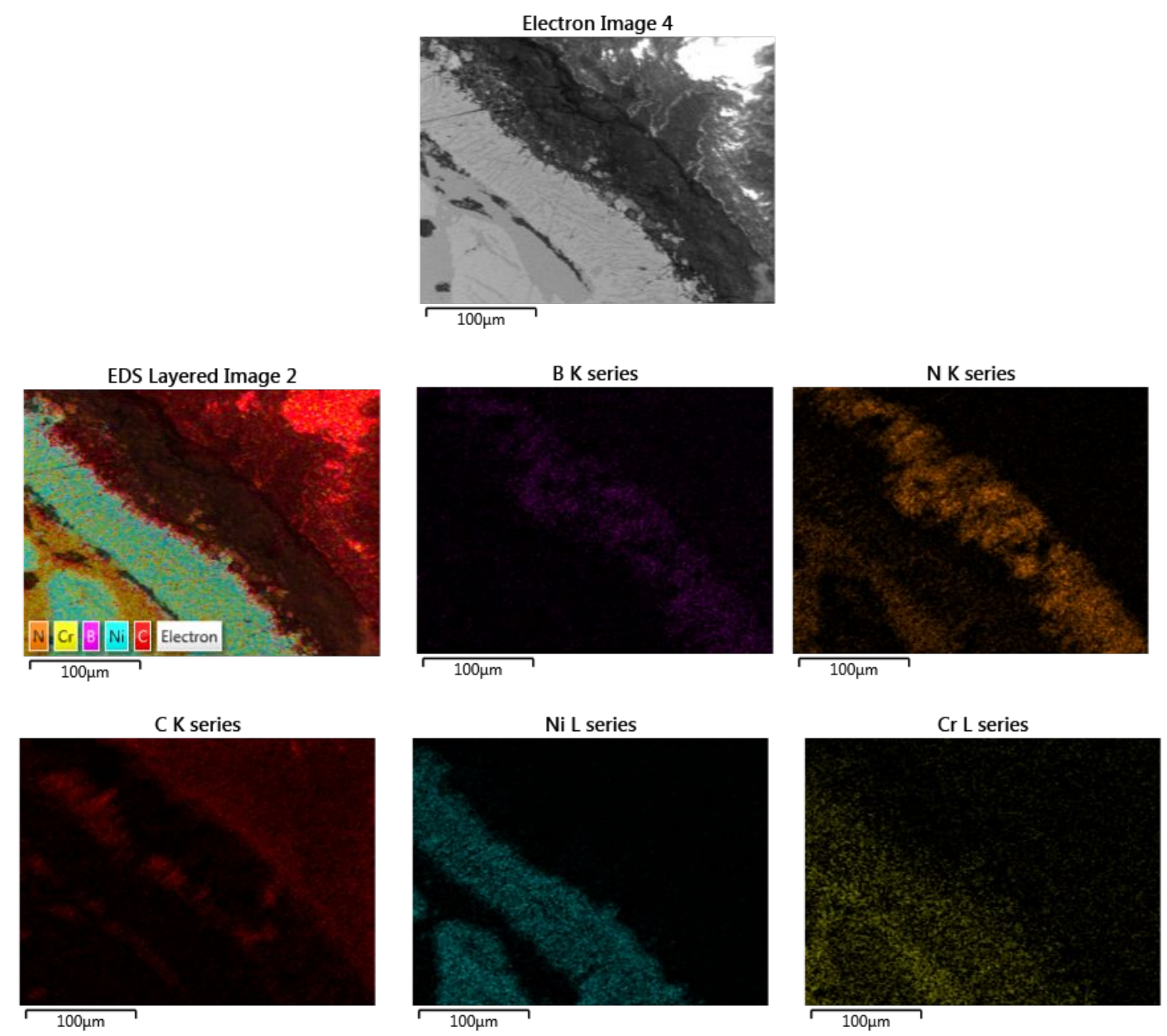

Figure s1. EDS mapping images of the alloy ingot cross-section at the carbon ratio of $0 \%$. Carbon signal at the interface comes from the polymer that used for embedding. 
Electron Image 9

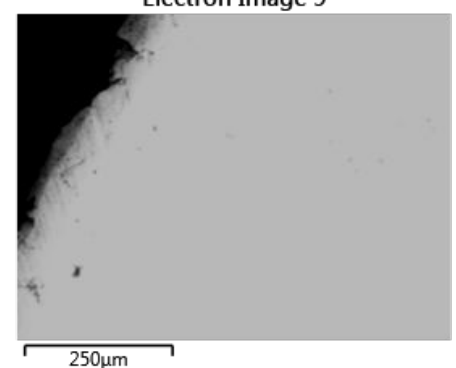

EDS Layered Image 4

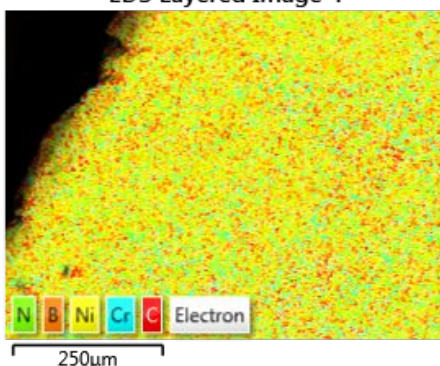

C K series
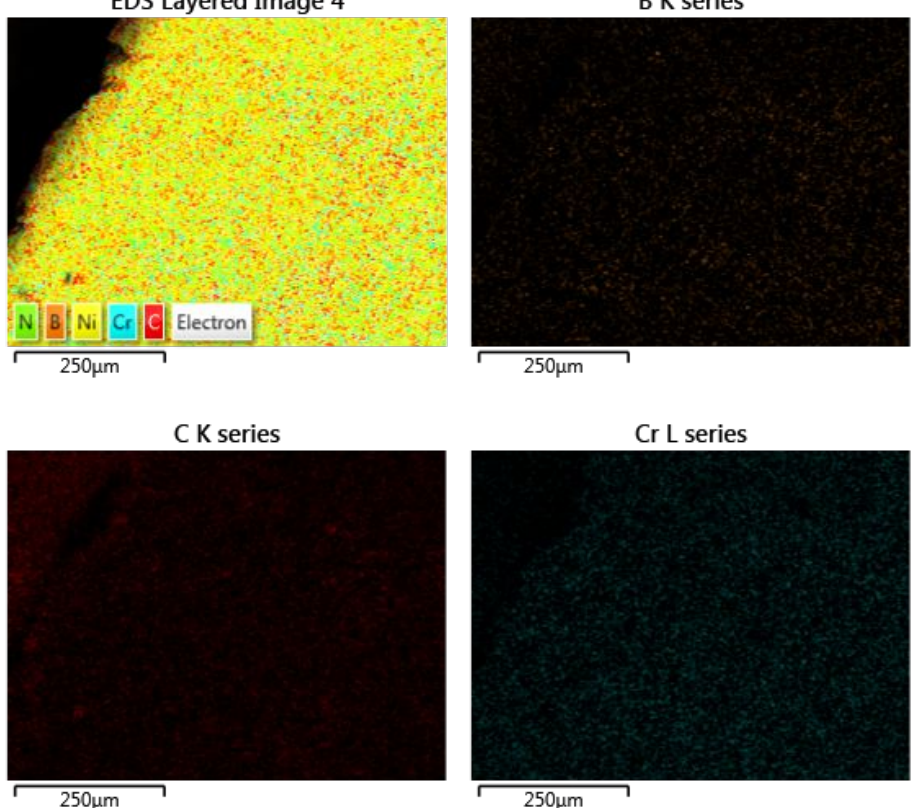

$250 \mu \mathrm{m}$
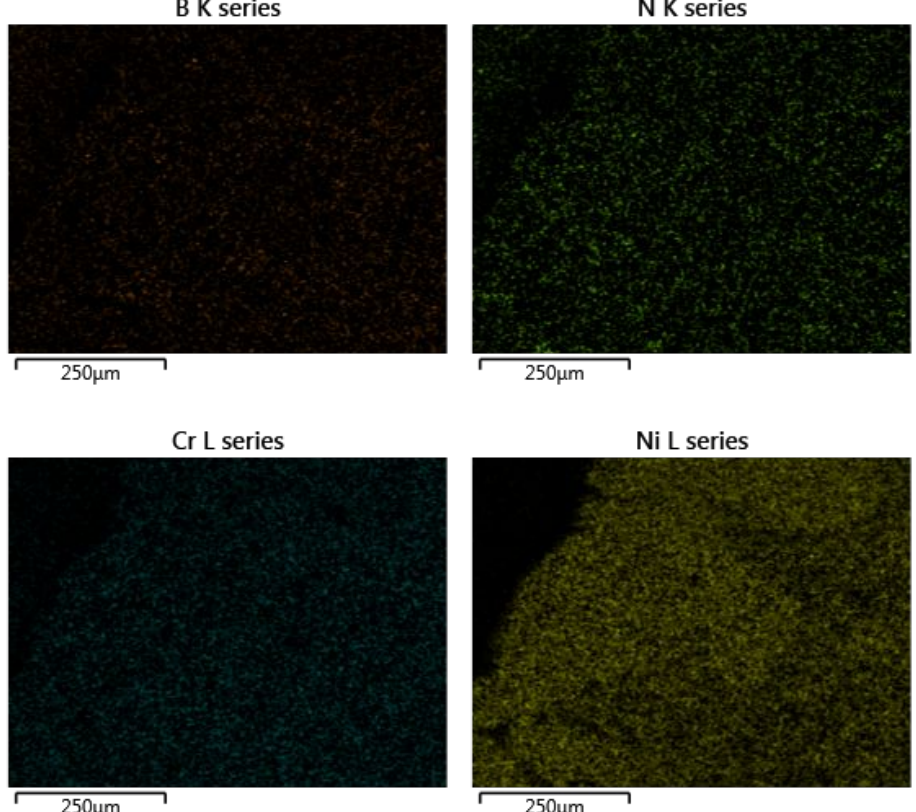

Ni L series

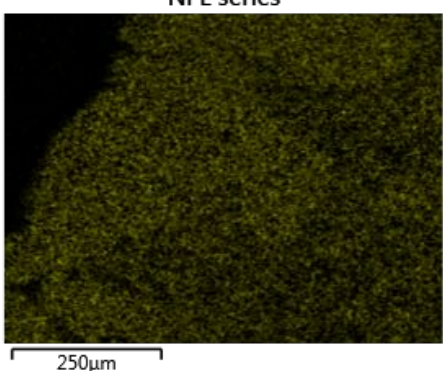

Figure s2. EDS mapping images of the alloy ingot section at the carbon ratio of $1.8 \%$. 